\title{
NUEVAS MIRADAS EN EL CINE LATINOAMERICANO: LAURA HUERTAS MILLÁN Y ANA VAZ
}

NEW LOOKS AT LATIN AMERICAN CINEMA:

LAURA HUERTAS MILLÁN AND ANA VAZ

DOI: $10.25100 /$ nc.v0i29.11601

Laura Dávila Argoty ${ }^{1}$

Pontificia Universidad Javeriana, Bogotá, Colombia.

laurava.94@gmail.com

ORCID: 0000-0002-6067-8484

Recibido: 10 de agosto de 2020

Aprobado: 3 de junio de 2021

ISSN en línea 2539-4355 / ISSN impreso 1900-9909 Este trabajo está bajo la licencia Creative Commons BY NC SA 4.0.

Dávila, L. (2021). Nuevas miradas en el cine latinoamericano: Laura Huertas Millán y Ana Vaz. Nexus, (29), 1-11. https://doi.org/10.25100/nc.v0i29.11601 
Resumen: El trabajo de las realizadoras Ana Vaz (Brasil) y Laura Huertas Millán (Colombia) reflexiona sobre los procesos históricos y culturales de América Latina. En su trabajo existe una recurrencia a temas relacionados a los procesos de colonización. Este artículo busca analizar ciertos elementos discursivos y formales presentes en las películas Viaje en tierra otrora contada (2011) y Há Terra! (2016) de Laura Huertas Millán y Ana Vaz, respectivamente. Con este propósito nos centraremos en la relación entre el territorio y las trazas de los procesos coloniales que se inscriben en este.

Palabras clave: Cine latinoamericano, Etnografía experimental, Colonización, Colonialismo.

Abstract: The work of the filmmakers Ana Vaz (Brazil) and Laura Huertas Millán (Colombia) reflects on the historical and cultural processes of Latin America. Thus, there is a recurrence of topics related to colonization processes in their work. This article seeks to analyze certain discursive and formal elements inside the films "Viaje en tierra otrora contada (Journey in a land once told)" (2011) and "Há Terra!" (2016) by Laura Huertas Millán and Ana Vaz, respectively. For this purpose, we will focus on the relationship between the territory and the traces of the colonial processes that are inscribed on this matter.

Keywords: Latin American cinema, Experimental ethnography, Colonization, Colonialism.

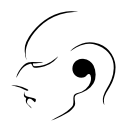

\section{Origen del artículo}

Este artículo surge de la ponencia presentada en el IX Seminario de Investigación en cine, organizado por el Festival Internacional de Cine de Cali (Cali, Colombia) realizado del 1 al 4 de diciembre de manera virtual. Asimismo, hace parte del proyecto de investigación en curso para el Máster en Estudios Cinematográficos y Audiovisuales de la Universidad Sorbonne Nouvelle - Paris 3. 
Ana Vaz (Brasil) y Laura Huertas Millán (Colombia) son dos realizadoras latinoamericanas. El trabajo de estas cineastas puede inscribirse dentro del campo de la etnografía experimental. Las películas de estas realizadoras tienen en común una reflexión de los procesos históricos y culturales del continente. En su trabajo existe una recurrencia a temas relacionados a los procesos de colonización. Tomando como partida el hecho de que a pesar de que estos procesos, en teoría, han finalizado, las huellas siguen presentes en las sociedades actuales y resuenan en el imaginario individual y colectivo. Siguiendo estas ideas este artículo busca analizar ciertos elementos discursivos y formales presentes en las películas Viaje en tierra otrora contada (2011) y Há Terra! (2016) de Laura Huertas Millán y Ana Vaz, respectivamente. Con este propósito, nos centraremos de manera más específica en el territorio y las trazas de los procesos coloniales que se inscriben en este. Este artículo hace parte de una investigación en curso que espera contribuir al análisis y creación de nuevos lenguajes cinematográficos que permitan cuestionar y dialogar con los discursos hegemónicos y los procesos históricos en América Latina.

La etnografía experimental pone en cuestión y critica ciertos aspectos ligados a la visión moderna de la antropología (Russell, 1999). El cine se convierte entonces en un dispositivo para reflejar nuestra relación con la realidad al mismo tiempo que un medio para cuestionarla. Según Catherine Russell (1999), la etnografía experimental no debe ser categorizada como un nuevo "género" cinematográfico sino como un útil crítico y estético de la representación. En este sentido, la etnografía experimental se configura como una incursión estética en la representación cultural, en donde se presenta una colisión entre la teoría social y la experimentación formal, existe entonces una confluencia entre vanguardia y etnografía. Esto permite replantearse la forma de filmar al igual que la relación con la alteridad (Russell, 1999). No se trata entonces de hablar por el Otro sino de hablar con, pensar con, como lo ha señalado la realizadora Trinh T. Minh-ha: "'speaking nearby' rather than 'speaking about'» (Chen, 1992). De esta forma, partiendo de estas películas es posible crear una interrogación del discurso y la representación colonial utilizada en el pasado para mostrar su presencia en el presente.

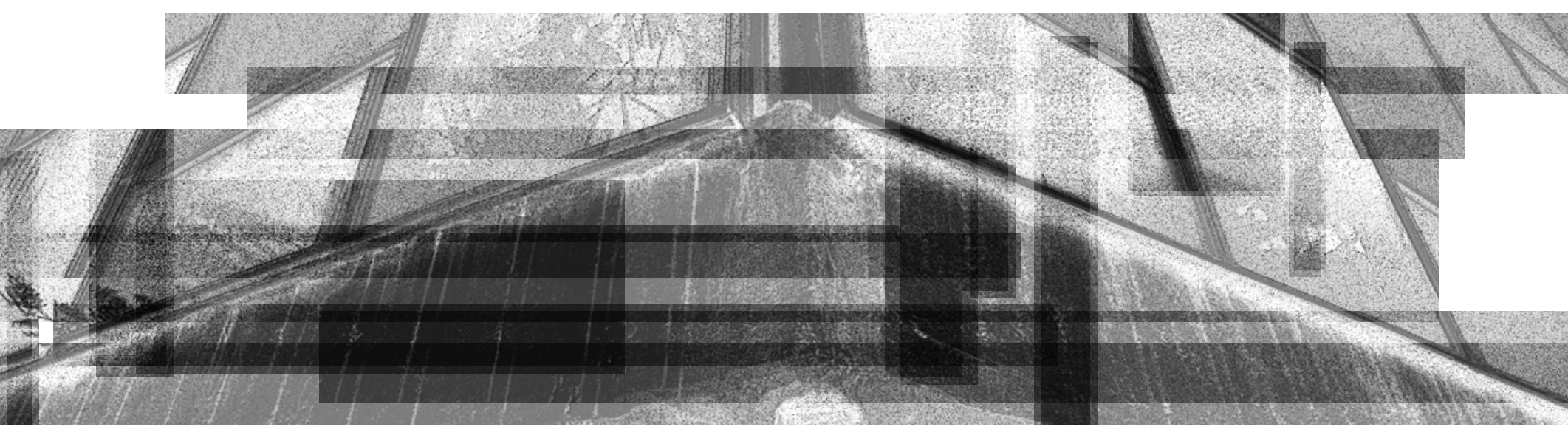


Si bien los procesos de colonización en América Latina comienzan con el "descubrimiento" del Nuevo Mundo a finales del siglo XV es a partir de los conceptos de "descubrimiento" y "Nuevo Mundo" que se desarrollarán una serie de procedimientos a través de los cuales diversas estructuras de dominación serán producidas y que además, contribuirán a la creación de una jerarquización de género y raza. Estructuras que continuarán siendo reproducidas a lo largo del tiempo, lo que nos permite decir que existe todavía una presencia de la colonialidad en nuestras sociedades. Como lo señala Rivera (2010) "estos horizontes recientes sólo han logrado refuncionalizar estructuras coloniales de larga data, transformándolas en formas de colonialismo interno" (p. 38). Un colonialismo interno, que corresponde al anclaje profundo del pasado colonial que prevalece en el presente.

Lo anterior se articula igualmente con las relaciones de apropiación, desposesión y explotación de la naturaleza que se establece, en primer lugar con los minerales - como el oro-y, después con las plantas, notablemente con las expediciones botánicas realizadas en América Latina durante el siglo XVIII y por las cuales "el mundo natural se incorpora al mundo de la propiedad" (Bowler, 1992, p. 14 citado por Nieto, 2019, p. 5). En consecuencia, la naturaleza es considerada como un recurso dispuesto a ser explotado. Es por esto que una serie de cuestionamientos se abren en relación a estos pueblos y territorios que fueron colonizados y donde estas lógicas colonizadoras continúan vigentes.

En este sentido, el cine de Laura Huertas Millán y su película Viaje a tierra otrora contada (2011) revisita estos hechos históricos. Huertas Millán es una cineasta y artista franco-colombiana. Ha desarrollado una serie en torno al exotismo a partir de procesos que tienen lugar en América Latina. En esta serie encontramos Sin dejar Huella (2009), Viaje a tierra otrora contada (2011) y Aequador (2012). Según relata Laura Huertas Millán, esta serie se comienza a pensar a partir de su encuentro con algunas fotografías en el Museo de Antropología de Chile. Se trata de fotos de los Mapuche en el Jardín de aclimatación de París a finales del siglo XX. Estas personas estaban siendo "exhibidas" como parte de lo que se llamó "Zoológicos Humanos". Estos fueron realizados desde 1870 hasta 1930 e intentaban recrear el entorno de los pueblos colonizados para ser mostrados en diferentes ciudades europeas.

La película Viaje en tierra otrora contada está rodada enteramente en el Jardín de Plantas de Lille, construido en 1948. Lugar que cuenta con una gran sala ecuatorial, en donde es posible encontrar diferentes plantas que fueron traídas desde América como resultado de las expediciones botánicas. Dichas expediciones se realizaron sobre todo en el siglo XVIII. Para esto, diferentes botánicos, al igual que artistas fueron enviados hacia América con el fin de «descubrir»nuevas plantas. Estas plantas fueron renombradas y cabe resaltar que los artistas que realizaban los dibujos botánicos eran entrenados visualmente para saber de qué manera debían plasmar las plantas, es decir, debían aprender a ver antes que dibujar la imagen. Su labor era captarlas en imagen tal como son, siguiendo instrucciones concretas de dibujo 
y siguiendo una serie de técnicas para transportarlas de forma debida a Europa. Por consiguiente, era necesario tener una mirada disciplinada que responda a los códigos de representación y reglas impuestas. Como afirma Nieto (2019) en su libro Remedios para el imperio, el hecho de nombrar la naturaleza, constituye entonces un gesto de poder y control, no solo de la naturaleza misma sino de las culturas. “Quien por primera vez reconoce un lugar, una planta o una medicina, proclama su derecho de posesión" (Nieto, 2019, p. 5).

Las plantas “descubiertas” en América Latina fueron rebautizadas por los naturalistas europeos, lo que supuso una apropiación no sólo de las plantas, sino también de la cultura y los conocimientos. Así, muchas de estas plantas circularon por diferentes epicentros europeos como París, Londres o Madrid. En otras palabras, esas plantas que fueron llevadas a Europa y que hoy forman parte de los jardines botánicos son un reflejo de la apropiación y el poder que se pretendía ejercer y mostrar a través de estas expediciones, de ese traslado de plantas, objetos y personas para ser exhibidos en las grandes ciudades de Europa, estableciendo un fuerte testimonio de poder y una forma de demostrar la hegemonía. Es necesario subrayar que estos procesos de apropiación constituyeron actos violentos no sólo con los habitantes de América Latina sino también con los otros no humanos, contribuyendo a la transformación de las prácticas y del paisaje. En palabras de Boumediene (2016):

\begin{abstract}
La apropiación que se observa en estas fuentes no es una "transferencia" o, peor aún, una "contribución” de América a Europa. Es un fenómeno disruptivo, que afecta a cada una de las configuraciones que atraviesa. Las prácticas cotidianas de cuidado, que permiten a las personas transformarse e interactuar con el mundo, son a su vez transformadas. Y todo lo que en ellas se basa en relaciones recíprocas y horizontales es capturado gradualmente por un proceso vertical: la colonización. (p. 22). ${ }^{2}$
\end{abstract}

En Viaje en tierra otrora contada, Huertas Millan nos traslada al pasado, a la colonización de América por medio del Jardín de Plantas, que se convierte un testimonio presente y vivo de este proceso pero que, como ella lo menciona, pareciera no poseer esas trazas. Aquí, no vemos estas capas de historia referentes a la colonización y violencia gracias a las cuales este lugar existe, como si de alguna manera estos sucesos permanecieran ocultos y se condenara al olvido. Es entonces cómo a través de esta película que Laura Huertas logra develar algunas de estas capas poco visibles pero que permanecen inscritas. Los procesos de colonización siguen presentes en nuestros cuerpos, en nuestra memoria, en nuestro paisaje y en nuestros territorios. Estas plantas que fueron llevadas a jardines botánicos y que aparecen en la película, son el reflejo de la apropiación y el poder que se buscó ejercer y mostrar a través de estas expediciones, de este traslado no solo de plantas sino también de objetos y personas para ser mostradas en las grandes ciudades de Europa. Estos espacios, como el Jardín de Plantas, se convierten entonces en un testimonio y prueba de las opresiones y los procesos de colonización que tuvieron lugar en América Latina y que

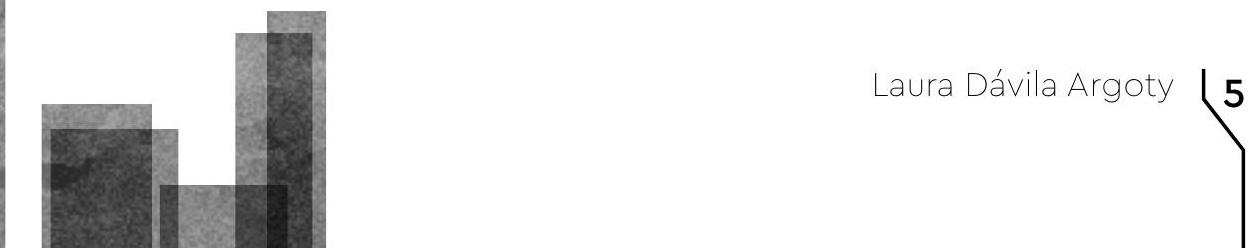


continúan hoy en día. La apropiación de estas plantas durante estas expediciones nos permite dar cuenta de la falta de comprensión de aquellos exploradores y colonizadores hacia lo que denominaron "Nuevo Mundo".

La planta no puede separarse -ni física ni metafísicamente- del mundo que la acoge. Es la forma más intensa, radical y paradigmática de estar en el mundo. La planta encarna el vínculo más estrecho y elemental que la vida puede establecer con el mundo. (...) Nunca podremos entender una planta sin entender el mundo. (Coccia, 2016, p. 18). ${ }^{3}$

En Viaje en tierra otrora contada, Huertas Millán usó diferentes textos escritos durante la conquista para hacer una adaptación libre que se escucha a través de una voz en off. De esta manera, estas historias son re-escritas, transformadas y re-imaginadas. Son traídas del pasado al presente. Entre estos textos encontramos títulos y autores como: Nus, feroces et antropophages de Hans Staden (Alemania, 1557); Historia verdadera de la conquista de Nueva España de Bernal Diaz del Castillo (España, 1632); Historia de un viaje hecho en la tierra de Brasil también conocida como América (Francia, 1578) de Jean de Léry y La misión de Ibiapaba del Padre Antonio Vieira (Portugal, 1657). La película inicia con el siguiente fragmento:

Habiéndome comprometido a trazar el mapa del curso del Amazonas -inicia- encontré una solución contra la inacción que una navegación tranquila me hubiera proporcionado. Debía estar continuamente pendiente de la brújula. Reloj en la mano, anotaba los cambios del curso del río, anotaba la altura meridiana del sol, también subía el barómetro. Me encontraba en el Nuevo Mundo. Alejado de todo comercio humano. En un laberinto de lagos de ríos y de canales que se adentran en todas direcciones hacia una selva inmensa que solo ellos hacen accesible. (Huertas, 2011, $0: 17)$.

Este texto creado por Huertas Millán funciona como dispositivo para hablar de la Amazonia y de las impresiones de los viajeros durante estas expediciones pero también para preguntarnos sobre las huellas coloniales inscritas en esta sala ecuatorial del Jardín de Plantas en Francia que, aunque es una suerte de artificio, es un testimonio evidente de la historia colonial. La realizadora ficcionaliza estas historias, estos discursos y es de esta manera que la película funciona no sólo como un dispositivo para cuestionar nuestros legados y las trazas coloniales, casi invisibles o poco discutidas, en Europa sino también como un diálogo en donde lo invisible deviene visible y en donde el espectador es invitado a dialogar y especular.

Por otra parte, encontramos el trabajo de la cineasta brasileña Ana Vaz. Su obra se caracteriza, de una parte, por la construcción de un lenguaje propio próximo en diferentes niveles a la etnografía y al cine experimental, y por el uso de archivos sonoros, fílmicos picturales; también por la presencia constante de las nociones de viaje y memoria. Las películas A Idade da Pedra (2013), Occidente (2015) y Há Terra! 
(2016) son para Vaz, la oportunidad de cuestionar la historia como relato oficial. En efecto, sus películas proponen una mirada que busca sobrepasar los discursos impuestos. En A Idade da Pedra vemos aparecer por primera vez a Ivonete dos Santos, quien reaparecerá en Há Terra! después de decidir unirse junto a su padre al Movimiento de los Trabajadores Rurales Sin Tierra ${ }^{4}$ en Brasil, movimiento social y político de más de 40 años que lucha por la distribución de las tierras improductivas.

En Há Terra! (2016) Vaz propone un lenguaje que se separa de una representación hegemónica de la cultura. Su cine se convierte en una herramienta para sobrepasar un tipo de representación que marginaliza otras historias. Para esto, utiliza una estética proveniente del cine experimental: movimientos aleatorios y apropiación de archivos sonoros, provenientes del film Francisca (1981) de Manoel de Oliveira. En efecto, al reutilizar estos archivos crea una intertextualidad con la película del realizador Portugués Manoel de Oliveira, creando un diálogo entre distintas épocas. Há Terra! Comienza con una cámara (operada por Vaz) que se mueve bruscamente generando barridos. Podríamos pensar que está buscando algo o alguien en medio de la naturaleza. A lo lejos, observamos a una niña corriendo que se esconde detrás de la hierba. La cámara, de hecho, la busca a ella, casi como en un juego de caza. Su película a menudo se describe como: "un encuentro, una caza, un relato diacrónico de mirar y devenir. Como en un juego, como en una persecución, la película erra entre personaje y tierra, tierra y personaje, depredador y presa" (Cinemateca de Bogotá, 2021).

Por un lado, la voz proveniente de la película del director portugués Manuel de Oliveira funciona como una evocación del pasado (la conquista de América) y una reafirmación del espacio donde se encuentra la joven. A lo largo de una película de Ana Vaz como la de Oliveira, la frase «Há terra!»Que podría traducirse como: «iTierra a la vista!» Funciona como una evocación que está ligada tanto al "descubrimiento" y la apropiación de tierras como al despojo de estas en la actualidad.

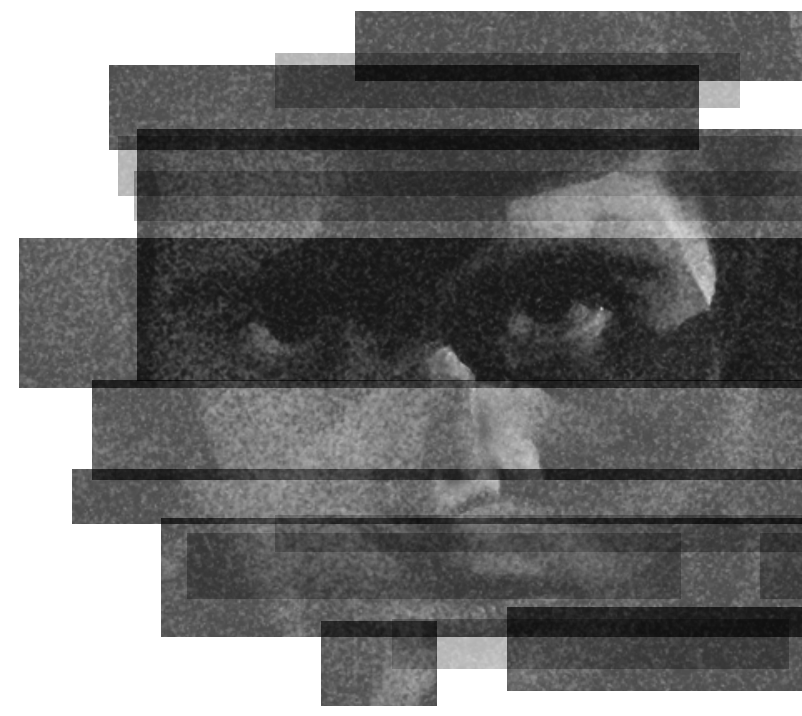
Citando a la crítica de cine Charlotte Garson en Festival du Cinéma du réel (2018) "la exclamación "Há Terra!" puede entenderse como la afirmación de que los sin tierra, organizados en movimiento durante cuarenta años, no deben ser privados de ella" ${ }^{5}$ En este sentido, esta joven se convierte a la vez en metáfora para nombrar el pasado y reivindicar las luchas que siguen estando presentes en la actualidad y que se conectan con su legado familiar. Por otra parte, la narración de esta película aparece a través de la voz off de Ivonete y ésta resuena en contraste con el discurso colonial. Esta historia cuenta un recuerdo. Es el recuerdo de cuando fue mordida por una culebra jararacussu ${ }^{6}$, cuando un día salió a recoger fruta con sus hermanos. Después de este evento, su pie se hincha y se desinfla según las fases de la luna. Es decir, el veneno de la serpiente reaparece con los ciclos lunares. 
Otro de los elementos que encontramos en Há Terra! son algunas pinturas de Mauricio Rugendas (1835), pintor y dibujante alemán que en su adolescencia aprendió a ser un pintor de batallas, lo que cambia con su llegada a Munich en donde empieza a inclinarse por la pintura de la naturaleza. Según cuenta Aira (2000) en su libro Un episodio en la vida del pintor viajero, el joven Rugendas llega a América Latina a la edad de 19 años y en 1821 comienza una expedición en Brasil que comprenderá las províncias de Rio de Janeiro, Minas Gerais, Mato Grosso, Espíritu Santo y Bahía. Varias de las pinturas y dibujos que realizó Rugendas en este viaje, y que aparecen en la película de Vaz, serán publicadas en 1835 en el libro Viaje Pintoresco a través del Brasil $7^{7}$ y gracias al cual entrará en contacto con Humboldt quien describirá a Rugendas como "creador y padre del arte de la presentación pictórica de la fisonomía de la naturaleza” (Aira, 2000, p. 12). Las pinturas de Rugendas presentes en HáTerra!, se centran en retratos de nativos, dibujos de plantas y paisajes. Algunas muestran la vida de los pueblos indígenas, así como de las comunidades negras: caza, enfrentamientos, la presencia del dominio colonial portugués y la esclavitud. Son por un lado, la ilustración de una visión exótica de América del Sur “descubierta” por los europeos y, por otro lado, el testimonio de los enfrentamientos violentos entre indígenas, esclavos y colonizadores.

Estos son algunos de los elementos que utiliza Ana Vaz para mostrar y complejizar la mirada del pasado colonial que aflora en el presente y que muestra cómo estas historias se siguen repitiendo. ¿Es posible pensar en el veneno que permanece en el cuerpo de la niña como una metáfora de los nuevos colonizadores que se arrojan aún hoy en día hacia las tierras de los otros? ¿Existe acaso un eterno devenir de las luchas y de los reclamos por el territorio?. Vaz graba en los mismos lugares en donde indígenas y esclavos fueron despojados, lugares en los que hoy continúan resistiendo y reclamando estos territorios. En este aspecto Ailton Krenak, líder y filósofo indigena brasileño, señala:

En el corazón de la historia brasileña está la idea de que los indios deberían haber contribuido al éxito del proyecto de las fuerzas coloniales de extenuación de la naturaleza. Sin embargo, el hecho de que resistieran, a pesar de las prácticas inhumanas que sufrían, manteniendo su modo de vida, no les permitió conservar sus territorios en su totalidad. Los indios alimentan la imaginación de muchos brasileños, pero no son bienvenidos en sus propias tierras.(Krenak \& Viveiros de Castro, 2020, p. 37)

En este sentido, la película no se limita a hacer una denuncia de dicho pasado sino que va más lejos. Há Terra! complejiza esta mirada y crea un espacio de reflexión que funciona, al mismo tiempo, como un reflejo de las circunstancias actuales de Brasil y América Latina llevándonos a lo que podría ser una "descolonización permanente del pensamiento" (Castro, 2010, p. 14). 
Para concluir, si bien estas dos películas tienen ciertos vectores comunes en cuanto a las trazas del colonialismo que siguen inscritas en nuestros territorios, uno de los rasgos especialmente interesante es el diálogo que establecen con estos procesos históricos y la pregunta por el territorio y la memoria. No hay miradas que se anulan, sino que por el contrario, se complejizan permitiendo multiplicar perspectivas. Asimismo, el hecho de crear un espacio de especulación que invita al espectador a reflexionar, permite que diferentes capas temporales aparezcan. Lo anterior, permite recuperar nuestra atención hacia estos temas que parecen quedar olvidados en la escritura de la Historia. Estas películas son una muestra de las capas de temporalidad que existen en un mismo territorio, por donde han pasado varias generaciones y diferentes procesos y en donde supuestamente estos procesos han finalizado. Sin embargo, las trazas siguen inscritas en el paisaje y en la memoria.
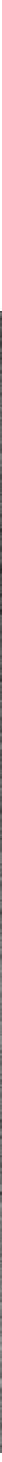


\section{Referencias}

Aira, C. (2000). Un episodio en la vida del pintor viajero (1. ${ }^{\text {a }}$ ed.). Beatriz Viterbo Editora.

Boumediene, S. (2016). La colonisation du savoir: une histoire des plantes médicinales du Nouveau Monde (1492-1750). Les Éditions des mondes à faire.

Castro, E. (2010). Metafísicas caníbales: líneas de antropología postestructural. Katz.

Chen, N. (1992). "Speaking Nearby:" A conversation with TrinhT. Minh-ha. Visual Anthropology Review, 8, 82-91. https://doi.org/10.1525/var.1992.8.1.82

Cinemateca de Bogotá (2021). Programa cortometrajes fronteras de la imagen. https: / / cinematecadebogota.gov.co/pelicula/programa-cortometrajes-fronteras-imagen

Coccia, E. (2016). La vie des plantes: une métaphysique du mélange. Éditions Payot \& Rivages.

Festival du Cinéma du réel. (2018, 26 de noviembre). Há terra! Cinéma du réel. https://www.cinemadureel.org/en/film/h-terra/

Krenak, A. \& Viveiros de Castro, E. (2020). Idées pour retarder la fin du monde. Editions Dehors.

Nieto, M. (2019). Remedios para el imperio: historia natural y la apropiación del Nuevo Mundo. Universidad de los Andes.

Rivera, S. (2010). Violencias (re)encubiertas en Bolivia. Piedra rota.

Rugendas, M. (1835). Voyage pittoresque dans le Brésil (M. de Golbéry, trad.). Engelman. https://gallica.bnf.fr/ark:/12148/bpt6k55677273.texteImage

Russell, C. (1999). Experimental ethnography: the work of film in the age of video. Duke University Press.

\section{Filmografía}

Huertas, L. (dir.). (2011). Viaje en tierra otrora contada [película]. Le Fresnoy.

Vaz, A. (dir.). (2016). Há terra! [película]. Marboeuf.

\section{Notas}

${ }^{1}$ Comunicadora Social con énfasis Audiovisual de la Pontificia Universidad Javeriana de Bogotá, y profesional en Cine y Audiovisual en la universidad Sorbonne Nouvelle - Paris 3, en donde actualmente cursa el Máster en Estudios Cinematográficos y Audiovisuales.

2 "L'appropriation observable dans ces sources n'est pas un "transfert" ou, pire, un "apport" de l'Amérique à l'Europe. Elle constitue un phénomène perturbateur, qui affecte chacune des configurations qu'il parcourt. Les pratiques quotidiennes du soin, celles qui permettent aux personnes de se transformer et d'interagir avec le monde, sont à leur tour transformées. Et tout ce qui en elles repose sur des rapports réciproques, horizontaux, se trouve peu à peu capturé par un processus vertical: la colonisation.”.

3 “On ne peut séparer — ni physiquement ni métaphysiquement — la plante du monde qui l'accueille. Elle est la forme la plus intense, la plus radicale et la plus paradigmatique de l'être-aumonde. La plante incarne le lien le plus étroit et le plus élémentaire que la vie puisse établir avec le monde. (...) Nous ne pourrons jamais comprendre une plante sans avoir compris ce qu'est le monde." 
${ }^{4}$ Movimiento político-social brasileño de inspiración marxista que lucha por la reforma agraria y la justicia social.

5 “l'exclamation « há terra! » (littéralement : « il y a (de) la terre ») peut aussi s'entendre comme l'affirmation que les sans-terre, non-possédants organisés en Mouvement depuis une quarantaine d'années, n'ont pas lieu d'en être privés.”.

${ }^{6}$ Especie de serpiente venenosa que habita en las selvas de América.

${ }^{7}$ Rugendas, J. M. (1835). Voyage pittoresque dans le Brésil / par Maurice Rugendas; traduit de l'allemand par M. de Golbéry. Engelman, Paris. https://gallica.bnf.fr/ark:/12148/bpt6k55677273. texteImage.

${ }^{8}$ Ce qui est au fondement de l'histoire du Brésil c'est l'idée que les Indiens auraient dû contribuer au succès du projet des forces coloniales d'épuisement de la nature. Qu'ils y aient résisté, malgré les pratique inhumaines qu'ils ont subies, en maintenant leurs formes de vie, ne leur a toutefois pas permis de conserver leurs territoires dans leur intégralité. Les Indiens nourrisent les imaginaires de nombreux brésiliens, mais ne sont pas les bienvenus sur leurs propres terres. 